\title{
ON JORDAN REPRESENTATIONS OF UNBOUNDED OPERATOR ALGEBRAS
}

\author{
SUBHASH J. BHATT
}

ABSTRACT. Every closed Jordan *-representation of an $E C^{*}$-algebra is the sum of a closed *-representation and a closed *-antirepresentation.

Atsushi Inoue [3] has recently initiated the study of a class of unbounded operator algebras, called $E C^{*}$-algebras. They seem to be quite useful in connection with unbounded Hilbert algebras. The purpose of this note is to prove the following theorem concerning the structure of unbounded $J^{*}$-representations of these algebras.

THEOREM. Every closed $J^{*}$-representation $\pi$ of an EC*-algebra $A$ is a direct sum of a closed ${ }^{*}$-representation $\pi_{1}$ and a closed ${ }^{*}$-antirepresentation $\pi_{2}$.

A mapping $\pi$ of a ${ }^{*}$-algebra $A$ with identity 1 into linear operators (not necessarily bounded) all defined on a common domain $D(\pi)$ dense in a Hilbert space $K$ is called a $J^{*}$-representation of $A$ on $K$ if, for all $x, y \in A, \alpha, \beta \in \mathbf{C}$ and $\xi, \eta \in D(\pi)$, the following hold:

1. $\pi(\alpha x+\beta y) \xi=\alpha \pi(x) \xi+\beta \pi(y) \xi$,

2. $\langle\pi(x) \xi, \eta\rangle=\left\langle\xi, \pi\left(x^{*}\right) \eta\right\rangle$,

3. $\pi(x) D(\pi) \subset D(\pi)$ and $\pi(x y+y x) \xi=\pi(x) \pi(y) \xi+\pi(y) \pi(x) \xi$,

4. $\pi(1)=I$.

The closure $\bar{\pi}$ of $\pi$ is a $J^{*}$-representation on $K$ defined as $\bar{\pi}(x)=\overline{\pi(x)}(x \in A)$, with domain $D(\bar{\pi})=\bigcap\{\overline{(\pi(x))} \mid x \in A\}$ where $D(\overline{\pi(x)})$ denotes the domain of the closure $\overline{\pi(x)}$ of the operator $\pi(x)$ in $K$. The map $\pi$ is closed if $\pi=\bar{\pi}$.

Our theorem is an extension of a result of Stormer [6, Theorem 3.3] on $C^{*}$-algebras. Let $A$ be an $E C^{*}$-algebra on a dense subspace $D$ of a Hilbert space $H$. Then its bounded part $\bar{A}_{b}=\{\bar{T} \mid T$ is a bounded operator in $A\}$ is a $C^{*}$-algebra. We apply Stormer's theorem to $\bar{A}_{b}$. Since $E C^{*}$-algebras are concrete realizations of locally convex $G B^{*}$-algebras [2, Theorem 7.11], the proof is completed by applying next a density theorem [1] viz. If $A$ is a $G B^{*}$-algebra with unit ball $B_{0}$, then the $B^{*}$-algebra $A\left(B_{0}\right)$ is sequentially dense in $A$. Note that $A\left(B_{0}\right)=\left\{\lambda x \mid \lambda \in \mathrm{C}, x \in B_{0}\right\}$ with the Minkowski functional $\|\cdot\|_{B_{0}}$ as the norm.

We shall need the following elementary lemmas.

Received by the editors September 29, 1980 and, in revised form, April 2, 1981.

1980 Mathematics Subject Classification. Primary 46L99.

Key words and phrases. $E C^{*}$-algebra, $J^{*}$-representation, $G B^{*}$-algebra.

() 1982 American Mathematical Society $0002-9939 / 81 / 0000-1030 / \$ 02.00$ 
Lemma A. Let $A$ be a locally convex $G B^{*}$-algebra with unit ball $B_{0}$. Let $\pi$ be a $J^{*}$-representation of $A$ on $K$. Then $x \rightarrow \overline{\pi(x)}$ defines a $J^{*}$-homomorphism of the $B^{*}$-algebra $A\left(B_{0}\right)$ into $B(K)$, the algebra of all bounded linear operators on $K$.

We omit the easy proof.

Lemma B. Let $A$ be a locally convex $G B^{*}$-algebra. Let $\tau$ be the largest locally convex $G B^{*}$-topology on $A$. Let $\pi$ be a $J^{*}$-representation of $A$ on $K$. Let $\sigma_{\pi}$ be the weak topology on $\pi(A)$ defined by the seminorms $\pi(x) \rightarrow|\langle\pi(x) \xi, \eta\rangle|$ for $\xi, \eta$ in $D(\pi)$. Then $\pi:(A, \tau) \rightarrow\left(\pi(A), \sigma_{\pi}\right)$ is continuous.

Proof. By the polarization identity, $\sigma_{\pi}$ is also determined by the seminorms $p(\pi(x))=|\langle\pi(x) \xi, \xi\rangle|$ for $\xi \in D(\pi)$. For any such $\xi$, let $f$ on $A$ be defined by $f(x)=\langle\pi(x) \xi, \xi\rangle$. Let $x \in A$. By [2, Proposition 5.1], $x^{*} x=h^{2}$ for some $h \geqslant 0$. Then $f\left(x^{*} x\right)=\left\langle\pi\left(h^{2}\right) \xi, \xi\right\rangle=\left\langle\pi(h)^{2} \xi, \xi\right\rangle=\|\pi(h) \xi\|^{2} \geqslant 0$. Thus $f$ is a positive linear functional on $A$ which by $[2, \S 8]$ is $\tau$-continuous. Now the result follows immediately.

Proof of THE THeOREM. Let $A$ be an $E C^{*}$-algebra over a domain $D$ dense in $H$, a Hilbert space. Let $\bar{A}=\{\bar{T} \mid T \in A\}$. Let $\sigma$ be the weak topology on $A$ defined by the seminorms $T \rightarrow p_{\xi, \eta}(T)=|\langle T \xi, \eta\rangle|$ for $\xi, \eta \in D$. Then [2, Theorem 7.12] shows that $(\bar{A}, \sigma)$ is a locally convex $G B^{*}$-algebra with unit ball $B_{0}=\{\bar{T} \in \bar{A} \mid\|T\| \leqslant 1\}$ so that the underlying $B^{*}$-algebra is $\overline{A_{b}}=\bar{A} \cap \beta(H)$. Let $\tau$ be the largest locally convex $G B^{*}$-topology on $A[2, \S 6]$. From now on, for the sake of simplicity, we omit writing the bar over the elements of $A$.

By Lemma A, $T \rightarrow \phi(T)=\overline{\pi(T)}$ defines a $J^{*}$-representation of $\overline{A_{b}}$ into $\beta(K)$. Let $B$ be the $C^{*}$-algebra in $B(K)$ generated by $\phi\left(\overline{A_{b}}\right)$. Hence [6, Theorem 3.3] there exist orthogonal central projections $E$ and $F$ in the von Neumann algebra generated by $B$ such that $T \rightarrow \phi_{1}(T)=\phi(T) E$ defines a ${ }^{*}$-representation of $\bar{A}_{b}$ on $K_{1}=E K$ and $T \rightarrow \phi_{2}(T) F$ defines a $*$-antirepresentation of $\overline{A_{b}}$ on $K_{2}=F K$. Also $E+F=I$. Let $D\left(\pi_{1}\right)=E D(\pi), D\left(\pi_{2}\right)=F D(\pi)$.

We show that, for each $T \in A, \xi, \eta \in D(\pi),\langle E \pi(T) \xi, \eta\rangle=\left\langle E \xi, \pi(T)^{*} \eta\right\rangle$. Indeed, by the density theorem, there is a sequence $T_{n}$ in $A_{b}$ such that $T_{n} \rightarrow T$ in $\tau$. By Lemma B,

$$
\begin{aligned}
&\langle E \pi(T) \xi, \eta\rangle=\langle\pi(T) \xi, E \eta\rangle=\lim _{n}\left\langle\pi\left(T_{n}\right) \xi, E \eta\right\rangle=\lim _{n}\left\langle E \pi\left(T_{n}\right) \xi, \eta\right\rangle \\
&=\lim _{n}\left\langle\pi\left(T_{n}\right) E \xi, \eta\right\rangle \quad \text { as } E \text { is central } \\
&=\lim _{n}\left\langle E \xi, \pi\left(T_{n}\right)^{*} \eta\right\rangle \\
&=\lim _{n}\left\langle E \xi, \pi\left(T_{n}^{*}\right) \eta\right\rangle \text { as } \pi\left(T_{n}^{*}\right) \subset \pi\left(T_{n}\right)^{*} \\
&=\left\langle E \xi, \pi\left(T^{*}\right) \eta\right\rangle \\
& \quad \text { again by Lemma B and the continuity of the involution } \\
&=\left\langle E \xi, \pi(T)^{*} \eta\right\rangle .
\end{aligned}
$$

This shows that $\eta \rightarrow\left\langle E \xi, \pi(T)^{*} \eta\right\rangle$ defines, for each $\xi \in D(\pi)$, a norm bounded linear functional on $D(\pi)$, and so $E \xi \in D\left(\pi(T)^{* *}\right)=D(\overline{\pi(T)})$. Thus 
$E \xi \in \bigcap\{D(\overline{\pi(T)}) \mid T \in A\}=D(\bar{\pi})=D(\pi)$. Thus $E D(\pi) \subset D(\pi)$. Similarly $F D(\pi) \subset D(\pi)$ and so $D\left(\pi_{1}\right)+D\left(\pi_{2}\right) \subset D(\pi), D\left(\pi_{1}\right)+D\left(\pi_{2}\right)=D(\pi)$.

Clearly $D\left(\pi_{1}\right)$ is dense in $K_{1}$ and $D\left(\pi_{2}\right)$ is dense in $K_{2}$. Let, for each $T \in A$, $\pi_{1}(T)=\pi(T) E, \pi_{2}(T)=\pi(T) F$ with domains $D\left(\pi_{1}\right)$ and $D\left(\pi_{2}\right)$ respectively. We show that $\pi_{1}$ and $\pi_{2}$ are the required maps.

Let $T \in A$. For each $n=1,2, \ldots$ let $T_{n}=T\left(1+\frac{1}{n} T^{*} T\right)^{-1}$. (Here the sum and the product are in the strong sense.) First we show that $T_{n} \rightarrow T$ in $\tau$.

$$
\begin{aligned}
& T-T_{n}=\frac{1}{n} T T^{*} T\left(1+\frac{1}{n} T^{*} T\right)^{-1} \\
&=\frac{1}{\sqrt{n}}\left(T T^{*}\right)\left(\frac{T}{\sqrt{n}}\right)\left(1+\left(\frac{T}{\sqrt{n}}\right)^{*}\left(\frac{T}{\sqrt{n}}\right)\right)^{-1} \in \frac{1}{\sqrt{n}}\left(T T^{*}\right) B_{0} \\
& \text { by }[5, \text { Theorem 13.13]. }
\end{aligned}
$$

Now by the separate continuity of multiplication in $\tau$, given an $o$-neighbourhood $V$, there exists an $o$-neighbourhood $U$ such that $T T^{*} U \subset V$. Further, as $B_{0}$ is $\tau$-bounded, $\sqrt{r} B_{0} \subset U$ for sufficiently small $r>0$. It follows that $T-T_{n} \in V$ eventually. Thus $T_{n} \rightarrow T$ in $\tau$.

Next we show that $\pi(A) D\left(\pi_{1}\right) \subset D\left(\pi_{1}\right)$ and $\pi(A) D\left(\pi_{2}\right) \subset D\left(\pi_{2}\right)$. Let $\xi, \eta \in D(\pi)$. By Lemma B,

$$
\begin{aligned}
\langle\pi(T) E \xi, \eta\rangle & =\lim _{n}\left\langle\pi\left(T_{n}\right) E \xi, \eta\right\rangle \\
& =\lim _{n}\left\langle E \pi\left(T_{n}\right) E \xi, \eta\right\rangle \quad \text { as } \pi\left(T_{n}\right) E \xi \in K \\
& =\lim _{n}\left\langle\pi\left(T_{n}\right) E \xi, E \eta\right\rangle \\
& =\langle\pi(T) E \xi, E \eta\rangle \quad \text { again by Lemma B } \\
& =\langle E \pi(T) E \xi, \eta\rangle
\end{aligned}
$$

and so $\pi(T) E \xi=E \pi(T) E \xi \in D\left(\pi_{1}\right)$. Hence $\pi(A) D\left(\pi_{1}\right) \subset D\left(\pi_{1}\right)$. Similarly $\pi(A) D\left(\pi_{2}\right) \subset D\left(\pi_{2}\right)$. Thus $\pi_{1}(T)$ and $\pi_{2}(T)$ are operators in $K_{1}$ and $K_{2}$ respectively.

Now given $T, S$ in $A$, again by the density theorem, there exist sequences $T_{n}$ and $S_{n}$ in $A$ such that $T_{n} \rightarrow T, S_{n} \rightarrow S$ in $\tau$. As $(A, \tau)$ is barrelled [2, Lemma 6.2], it is hypocontinuous by [2, Lemma 6.3]. Since the multiplication in a hypocontinuous algebra is easily see to be sequentially jointly continuous, $T_{n} S_{n} \rightarrow T S$. Then for each $\xi, \eta$ in $D\left(\pi_{1}\right)$, repeated uses of Lemma B give

$$
\begin{aligned}
\left\langle\pi_{1}(T) \pi_{1}(S) \xi, \eta\right\rangle & =\lim _{n} \lim _{k}\left\langle\pi_{1}\left(T_{n}\right) \pi_{1}\left(S_{k}\right) \xi, \eta\right\rangle \\
& =\lim _{n}\left\langle\pi_{1}\left(T_{n}\right) \pi_{1}\left(S_{n}\right) \xi, \eta\right\rangle \\
& =\lim _{n}\left\langle\pi_{1}\left(T_{n} S_{n}\right) \xi, \eta\right\rangle \text { as } \phi_{1} \text { is a representation } \\
& =\left\langle\pi_{1}(T S) \xi, \eta\right\rangle .
\end{aligned}
$$

Thus $\pi_{1}$ is a ${ }^{*}$-representation. Similarly $\pi_{2}$ is a ${ }^{*}$-antirepresentation.

It only remains to show that each of $\pi_{1}$ and $\pi_{2}$ is closed. Let $T \in A$ and $\xi \in D\left(\overline{\pi_{1}(T)}\right)$, the domain of the closure in $K$. Then for some sequence $\left\{\xi_{n}, \pi_{1}(T) \xi_{n}\right\}$ 
in the graph of $\pi_{1}(T), \xi_{n} \rightarrow \xi$ in $K_{1}$. Then clearly $\xi \in D(\overline{\pi(T)})$ and so $\xi \in D(\bar{\pi})=D(\pi)$. Also $\pi_{1}$ is closed, and similarly so is $\pi_{2}$.

This completes the proof of the theorem.

Note that what is essentially required in the proof is the fact that $A$ is a locally convex $G B^{*}$-algebra. Hence [4, Corollary 3.4] immediately gives the following.

COROLlaRY. Let $D$ be a pure unbounded Hilbert algebra over a maximal unital Hilbert algebra $D_{0}$. Then each closed $J^{*}$-representation of $D$ is the direct sum of $a$ closed $*_{\text {-representation and a closed }}^{*}$-antirepresentation.

\section{REFERENCES}

1. S. J. Bhatt, $A$ note on generalized $B^{*}$-algebras, J. Indian Math. Soc. (to appear).

2. P. G. Dixon, Generalized B*-algebras, Proc. London Math. Soc. (3) 21 (1970), 693-715.

3. A. Inoue, On a class of unbounded operator algebras. I, II, III, Pacific J. Math. 65 (1976), 38-57; 66 (1976), 411-431; 69 (1977), 105-115.

4. Unbounded Hilbert algebras as locally convex *-algebras, Math. Rep. Kyushu Univ. 10 (1976), 113-129.

5. W. Rudin, Functional analysis, 2nd ed., McGraw-Hill, New York, 1973.

6. E. Størmer, On the Jordan structure of $C^{*}$-algebras, Trans. Amer. Math. Soc. 120 (1965), 438-447.

Department of Mathematics, Sardar Patel University, Vallabh Vidyanagar-388120, India 